\title{
A Study of Benefits in Object Based Storage Systems
}

\author{
Clarence J M Tauro \\ Center for Research \\ Christ University \\ Bangalore, India
}

\author{
N Ganesan \\ Director (MCA) \\ RICM, Bangalore
}

\author{
Anjan Kumar RS \\ Dept. of Computer Science \\ Christ University \\ Bangalore, India
}

\begin{abstract}
The fundamental storage component in Object based storage system (OBS) is an Object [5]. Object interface of OBS provides prominent storage service and metadata abstraction to various application classes on single platform. The Object interface also offers performance gain and security features in storage systems [6]. This paper focuses on the benefits involved in OBS such as native security, scalability and dynamic reconfiguration.
\end{abstract}

\section{Keywords}

OBS, Object, Storage

\section{INTRODUCTION}

The storage systems are having different groups of users and different workloads. They are also having different characteristics and priorities. Enabling the multiple applications on a single platform for Object storage is very desirable. Object, In an Object based storage system defines various aspects of data along with the set of attributes. OBS will be aware of different application class and provides resource based allocation

OBS allows storage devices (whether individual disc drives, disc arrays or other storage controllers) to explicitly manage space allocation, a function traditionally performed in the host file system or database software[2]. OBS also provides for strong security protection enforced by storage devices, each request must be explicitly authorized. The OBS allows attributes to be associated with data objects. These attributes can give the storage device detailed information on the characteristics of a particular data object: how it is to be handled and how it will be used.

Security is perhaps the single most important feature of object-based storage that distinguishes it from block-based storage. Objects make it possible to partition large devices or fabrics into multiple security domains whose access policies are individually determined by the storage applications.

\section{BENEFITS OF OBS}

\subsection{Storage Management}

Objects really make the self-management of storage possible. Space can be efficiently managed when OBS has access to attribute management.

An example, initially system backup was taken during idle hours; this would be typically done on the weekend to avoid any hindrance to actual work. But however over a period of time the data backup has become a continuous process, this means a large volume of data say Terabytes of data are captured continuously. By taking action on an object based attributes assigned to it, The OBS could inform a backup function whenever an object has reached the correct state for its backup to be taken.

Data integrity becomes primary concern when backup data grows in size, the backup of all files could be spread over period - during which others are still being updated.

The OSD storage management component lays emphasis on mapping spatial object to the physical organization in the storage media, and ensures to allocate storage capacity for individual data entities and managing free space [3].

\subsection{Intelligence}

Object based storage has enabled the storage devices to actively learn important characteristics of the environments in which they operate [10]. With objects, storage devices would understand some of the relationships between the blocks on the device, and can use this information to organize the data better.

- Object attributes can be of different forms:

- Static information about the object like Creation time

- Dynamic information which is updated every time when accessed like last accessed time

- Information specific to storage application like filename

- Information specific to current user like Quality of Service

Finally, Objects enable storage devices to become aware of the environments in which they operate, and thereby better allocate and provision resource [7]

\subsection{Scalability}

The Association of Storage Networking Professionals (ASNP) estimates that there were over 1 million full-time or part-time storage administrators in 2004.

Most of the companies listed in Fortune are known to approaching 1 petabyte of data. Industrial research labs like British Petroleum (BP) use 600 terabytes for petroleum discovery [1]. To implement this system, multiple levels of abstraction and management are required; each of this must be configured and operated individually. 
It is difficult to manage the metadata, as it spread throughout the system. With the help of Object-Based Stored Devices (OSD) interface, metadata could be associated and stored directly with each object and it is automatically carried between layers across devices. Space management metadata - which blocks belong to which objects - becomes the responsibility of the storage device.

Object-Based Stored Devices (OSD) in a system allow metadata layers to be collapsed, this in turn improves the scalability of the system. This also reduces the processing requirements and overhead, a single server manages a larger number of devices than with traditional block-based interfaces. This allows users to build storage clusters for very large installations or to use more economical systems than is possible with block-based storage.

\subsection{Security}

The defense-in-depth security at lowest system levels, like disc drives are enabled by OSD interface in OBS. With an OSD interface, the individual disc drive would be able to validate each read and write request. The protection of user data and storage is taken care by improved granularity of Object level security.

For Each request a logical check is performed to gain access to user data by OSD. A credential must be provided by a system and this needs to validate to gain access to OSD devices. This check prevents both accidental access for undesirable requests and also prevents malicious access for unauthorized requests (from hackers either internal or external.

A group of Massachusetts Institute of Technology (MIT) researchers conducted a test around one hundred disc drives and found data which is never left accessible.

Various disc drives like Small Computer System Interface (SCSI) and Advanced Technology Attachment (ATA) of different sensitive applications has been formatted and they are able to retrieve the data rarely. The earlier network storage architecture, provides access to all data and metadata via metadata server, this ensures centralized security control of data. [1]

The separation of Client and OSD in OBS architecture provides the following benefits:

- Network security against attacks are evaded

- Authentication is enabled between the client and OSD mutually

- The communications are encrypted among the client and OSD [3]

\subsection{Dynamic Reconfiguration}

Metadata is carried between and across the layers of storage systems and also associated with data object via OSD interface[8]. The system layer enables the fragmentation number of attributes for individual object. When Object is passed via system layer it performs the action based on the values of attributes associated with the system. Any other attributes which are unmodified wouldn't be acted upon. This means the objects marked as High-reliability can be treated differently than objects marked Temporary [9].

A system invariably moves data objects between storage devices or provide data-specific functionality, because storage devices are aware of data objects and management attributes are associated with data objects. During migration the metadata is intact between devices. The ability to associate semantic metadata directly with objects makes it possible for storage devices to flexibly manage the data in the object for performance and reliability.

\subsection{Heterogeneous Computing using OBS}

The objective of Object Based Storage is to enable the sharing of storage in a heterogeneous processor cluster. Operating system metadata structures are unique.

Thus OBS have the possibility to have common platform on which any OS can overlay its file system. This ensures hostindependent concept of object storage [4].

\section{RELATED WORK}

The primary work on object-based storage occurred at Carnegie Mellon University's Parallel Data Lab (PDL) with the Network-Attached Secure Disks (NASD) project [1]. This research is primarily was focused on adding processing power to individual disks in cost effective manner, specifically designed for networking security and basic space management functionality

This research led to a larger industry-sponsored project under the auspices of the National Storage Industry Consortium (NSIC). Several storage companies joined the collaboration, and NASD was generalized to network-attached storage devices, where individual drives, array controllers, and appliances could take advantage of the interface change.

\section{SUMMARY}

Block based interface though as made substantial advances in storage devices, but the Object interface offers storage which is secure and easy to share across platforms, none the less it also provides high performance. Objects provide the storage device with a cognizance of the storage application and enable more intelligence in the device. OBS also exploits the increasing capabilities of storage devices.

\section{ACKNOWLEDGMENTS}

We are heartily thankful to Prof. Jibrael and Prof. Joy Paulose, Department of Computer Science, Christ University, whose encouragement, guidance and support enabled us to develop an understanding of the subject.

\section{REFERENCES}

[1] Mesnier, M.; Ganger, G.R.; Riedel, E.; , "Object-based storage," Communications Magazine, IEEE , vol.41, no.8, pp. 84- 90, Aug. 2003

[2] Whitepaper: Seagate Technology Paper "The Advantages of Object-Based Storage" www.seagate.com/docs/pdf/whitepaper/tp_536.pdf

[3] Zhongmin Li; Zhanwu Yu; , "A Credential-based Security Mechanism for Object-based Storage," Communications, Circuits and Systems Proceedings, 2006

[4] Whitepaper: Seagate Technology Paper "Seagate Technology" ftp://ftp.t10.org/t10/document.99/99315r1.pdf

[5] Ling-Fang Zeng; Dan Feng; Ling-Jun Qin; , "SOSS: smart object-based storage system," Machine Learning and Cybernetics, 2004. Proceedings of 2004 International Conference on , vol.5, no., pp. 3263- 3266 vol.5, 26-29 Aug. 2004 
[6] Qingsong Wei; Zhixiang Li; , "DifferStore: A differentiated storage service in object-based storage system," Cluster Computing, 2008 IEEE International Conference on , vol., no., pp.185-193, Sept. 29 2008-Oct. 12008

[7] Meng Lingkui; Lou Shurong; Xie Wenjun; Zhang Wen"Object-Based Spatial Information Storage for WEBGIS," Education Technology and Training, 2008

[8] Dan Feng; Fang Wang; Qun Liu; , "Adaptive Policy Trigger Mechanism for OBSS," AINA 2005 IEEE International Conference on, vol., no., pp.591-595, March 28 2005-30-March 2005
[9] Gongye Zhou; Lanlan Yuan; Jincai Chen; ," B+ Tree Management Method of Object Attributes for ObjectBased Storage," Networking, Architecture and Storage 2007 IEEE International Conference on , vol., no., pp.253-254, 29 July 2007-31 July 200

[10] Zhipeng Tan; Yanli Yuan; Dan Feng; Tian Zhan; , "Implementation of method object in active object-based storage systems," Cloud Computing and Intelligence Systems (CCIS), 2011 IEEE International Conference on, vol., no., 204-211, Sept. 15 2011-Sept. 172011 